\title{
Bicuspid aortic valve
}

\section{Background}

The aortic valve of the heart opens and closes in a coordinated fashion to ensure that oxygen-rich blood is pumped efficiently from the heart to the body. The aortic valve normally consists of three leaflets or cusps, however approximately 1-2\% of the population have only two cusps, this is known as a bicuspid aortic valve. The two cusps of a bicuspid aortic valve are usually unequal in size, which causes turbulent blood through the valve, increasing the likelihood of calcium deposition on the valve, which can cause narrowing (known as stenosis) or leakage of the valve (known as regurgitation or insufficiency). Individuals with a bicuspid aortic valve require close monitoring to prevent the development and progression of complications, prolonging survival and improving the quality of life of those affected.

\section{Sign and symptoms}

There are often no symptoms for many years. When calcium deposits around the valve progressively cause the valve to stiffen, the valve may narrow, which is known as aortic stenosis. This can cause chest pain on exertion, shortness breath, fatigue, dizziness and fainting. Inadequate closure of a bicuspid aortic valve may cause leakage of blood back into the heart, known as aortic regurgitation or insufficiency. In this situation, the heart must work harder as the valve leaks after each contraction.

\section{Complications}

For individuals with aortic stenosis or regurgitation, the heart must work harder to ensure a sufficient blood is pumped round the body. Over time, the heart becomes unable to pump sufficient blood to meet the body's needs, resulting in heart failure. Those with a bicuspid aortic valve are also at increased risk of valvular infection, known as infective endocarditis. This can be life threatening and require prolonged courses of antibiotics.

Individuals with bicuspid aortic valve are at an increased risk of developing dilatation of the first part of the aorta, an ascending aortic aneurysm. Ascending aortic aneurysm puts the individual at risk of two important complications: aortic dissection and aortic rupture. An aortic dissection occurs when a tear develops in the inner layer of the aorta (the intima) which creates an alternative channel for blood to flow which can occlude the blood supply to vital organs and put the patient at risk of aortic rupture.

\section{Investigations}

The most useful investigation for diagnosis of a bicuspid aortic valve is a transesophageal echocardiogram (TEE), which generates dynamic ultrasound images of the heart via a probe inserted into the esophagus. TEE can detect the presence and severity of complications such as aortic stenosis and regurgitation. Other valuable tests include electrocardiography, which detects the electrical activity of the heart, and CT scans of the heart and aorta to detect the presence of aneurysms or dissections.

\section{Management}

Generally aortic valve replacement is necessary for bicuspid valve disease with severe stenosis or regurgitation. Replacement valves may be engineered from mechanical or bioprosthetic materials. Mechanical valves tend to be more durable with a lower risk of re-operation later in life, but require lifelong anti-clotting medications (warfarin) to prevent blood clots forming on the valve. As with all major heart operations, there are a number of possible complications from replacement of the aortic valve and root, including bleeding, wound infection, heart attack, stroke and a small risk of death. The benefits are to reduce symptoms, improve survival, improved quality of life and to prevent the progression of complications secondary to the bicuspid valve. The risks of each surgical option should be weighed against the benefits and discussed with your surgeon.

For more information, please visit the following websites: https://my.clevelandclinic.org/health/articles/bicuspidaortic-valve-disease http://columbiasurgery.org/conditions-and-treatments/ bicuspid-valve-disease

https://emedicine.medscape.com/article/893523-overview

doi: 10.21037/acs.2017.11.07

Section Writer: Christopher Harris

Illustration Editor: Beth Croce

Section Editor: Stine Munkholm-Larsen
For specific information concerning your medical condition, ACS suggests that you consult your physician. This page may be photocopied non-commercially by physicians to share with patients. 